TRANSACTIONS OF THE

AMERICAN MATHEMATICAL SOCIETY

Volume 350, Number 10, October 1998, Pages 4253-4264

S $0002-9947(98) 02011-\mathrm{X}$

\title{
CHUNG'S LAW FOR INTEGRATED BROWNIAN MOTION
}

\author{
DAVAR KHOSHNEVISAN AND ZHAN SHI
}

\begin{abstract}
The small ball problem for the integrated process of a real-valued Brownian motion is solved. In sharp contrast to more standard methods, our approach relies on the sample path properties of Brownian motion together with facts about local times and Lévy processes.
\end{abstract}

\section{INTRODUCTION}

Let $\{X(t) ; t \in T\}$ be a continuous centered Gaussian process taking values in $C(T)$ - the space of all real-valued continuous functions from $T$ into $\mathbb{R}$. Given a seminorm $\|\cdot\|$ on $C(T)$, the small ball problem for $X$ is a determination of the rate of explosion of the following as $\varepsilon \rightarrow 0^{+}$:

$$
-\log \mathbb{P}(\|X\|<\varepsilon)
$$

This problem has a long history and little is known in general. One cannot always hope to see an explicit evaluation of (1.1) from the covariance function of $X$. Even the estimation of the rate of explosion in (1.1) is usually a difficult problem. Indeed, as can be surmised from Kuelbs and $\mathrm{Li}[16]$, this problem is often equivalent to open problems in functional analysis. Known results for well-behaved processes (especially, Brownian motion and related processes) can be found for example in the recent works [4], [7], [15]-[18], [21]-[23], [27]-[31].

The aim of this paper is to study the special case of integrated Brownian motion. Let $\{W(t) ; t \geq 0\}$ be a one-dimensional Brownian motion starting from 0 , and define

$$
X(t) \triangleq \int_{0}^{t} W(s) d s, \quad t \geq 0
$$

(There is no loss in restricting attention to one dimension.)

There are several compelling reasons behind the present work which we list below.

(a) Existing techniques in the literature do not seem to yield a satisfying answer to the small ball problem for this example (cf. Remark 2.1 below).

(b) Integrated Brownian motion is an interesting process on its own. For example, it appears naturally in classical literature in mechanics and biology ([10], [24],

Received by the editors October 19, 1996 and, in revised form, January 3, 1997.

1991 Mathematics Subject Classification. Primary 60G15, 60J65; Secondary 60J55.

Key words and phrases. Small ball probability, integrated Brownian motion.

Research partially supported by grants from the National Science Foundation and the National Security Agency.

(C)1998 American Mathematical Society 
[26]; cf. Lachal [19] for a detailed account), as well as in the more recent works in quantum probability ([1], [2], [14]).

(c) Our analysis of $X$ uses a genuinely new approach to the small ball problem. We first prove a Chung's law of the iterated logarithm (LIL) for $X$ and then use it to establish the rate of explosion in the small ball problem. This is in reverse order to the standard approach. Moreover, our approach uses local times and properties of Lévy processes; a method which may be of independent interest.

For notational convenience, we write throughout the paper,

$$
X^{*}(t) \triangleq \sup _{0 \leq s \leq t}|X(s)|, \quad t \geq 0,
$$

where the process $X$ is defined in (1.2). The main result of the paper is the following small ball estimate.

Theorem 1.1. There exists a universal constant $c_{1} \in(0, \infty)$ such that

$$
\lim _{\varepsilon \rightarrow 0} \varepsilon^{2 / 3} \log \mathbb{P}\left(X^{*}(1)<\varepsilon\right)=-c_{1} .
$$

Not surprisingly, Theorem 1.1 is related to a Chung-type LIL which is stated as follows.

Theorem 1.2. Let $c_{2} \triangleq\left(c_{1}\right)^{3 / 2}$, with $c_{1}$ defined in (1.4). Then

$$
\liminf _{t \rightarrow \infty} \frac{X^{*}(t)}{\varphi(t)}=c_{2}, \quad \text { a.s. }
$$

where

$$
\varphi(t) \triangleq \frac{t^{3 / 2}}{(\log \log t)^{3 / 2}} .
$$

Taking the $L^{2}$-norm instead of the sup-norm in (1.1), the small problem becomes far more treatable. We include the following explicit results for the sake of completeness.

Theorem 1.3. We have

$$
\begin{aligned}
\lim _{\varepsilon \rightarrow 0} \varepsilon^{1 / 3} \log \mathbb{P}\left(\int_{0}^{1} X^{2}(s) d s<\varepsilon\right) & =-\frac{3}{8}, \\
\liminf _{t \rightarrow \infty} \frac{\int_{0}^{t} X^{2}(s) d s}{t \varphi^{2}(t)} & =\frac{27}{512}, \quad \text { a.s. }
\end{aligned}
$$

Remark 1.4. Since $X^{*}(1) \geq \sqrt{\int_{0}^{1} X^{2}(s) d s}$, it follows from (1.7) that $c_{1} \geq 3 / 8$. The exact value of $c_{1}$ is unknown.

Let us say a few words about the proof of Theorem 1.1. It is easy to see that the limit in (1.4) exists (therefore $c_{1}$ is well-defined) and is moreover (strictly) negative. The harder part is to verify that $c_{1}<\infty$. We are not able to show this by a direct estimation of the small ball probability. Rather, we first show (1.5) and then use it to show that $c_{1}<\infty$. This is probably the most striking feature in the analysis of the example. The idea is to consider the inverse local time at 0 of $W$. Brownian motion integrated up to the inverse local time is a "well-behaved" Lévy process (say, $Y$ ). We will show that the absolute maximum process of $Y$ is identical to $X^{*}$ 
taken at the inverse local time. Once Theorem 1.2 is obtained, it is not hard to deduce the small ball probability via the Borel-Cantelli lemma.

Theorems 1.1 and 1.2 are proved in Section 2, and Theorem 1.3 in Section 3. Some further remarks are presented in Section 4.

\section{Proofs of Theorems 1.1 And 1.2}

The proofs of Theorems 1.1 and 1.2 are carried out in five steps:

(a) the limit in (1.4) exists and is negative;

(b) the lower limit in (1.5) is a (possibly degenerate or infinite) constant, which will be denoted by $c_{2}$;

(c) $c_{2}<\infty$;

(d) $c_{2}>0$ and the limit in (1.4) is finite;

(e) $c_{2}=\left(c_{1}\right)^{3 / 2}$.

We briefly mention the essential ingredients in each of the five steps: (a) subadditivity; (b) 0-1 law; (c) introduction of inverse local time and Lévy process techniques; (d)-(e) quasi-equivalence between Chung's LIL and the small ball estimate.

2.1. Existence and strict negativity of the limit in (1.4). First, it is worth noting that the process $X$ defined in (1.2) inherits the Brownian scaling property, in the sence that, for any fixed $t>0$,

$$
X(t) \stackrel{(\mathrm{d})}{=} t^{3 / 2} X(1), \quad \text { and } \quad X^{*}(t) \stackrel{(\mathrm{d})}{=} t^{3 / 2} X^{*}(1),
$$

where " $\stackrel{(\mathrm{d})}{=}$ " denotes identity in distribution. Hence,

$$
\mathbb{P}\left(X^{*}(1)<\varepsilon\right)=\mathbb{P}\left(X^{*}\left(\varepsilon^{-2 / 3}\right)<1\right) .
$$

By the monotonicity of $t \mapsto X^{*}(t)$, it suffices to verify that

$$
\lim _{n \rightarrow \infty} \frac{1}{n} \log \mathbb{P}\left(X^{*}(n)<1\right)
$$

exists and is negative (the proof of the finiteness is postponed to Section 2.4). To this end, let

$$
a_{n} \triangleq \sup _{x \in \mathbb{R}} \mathbb{P}\left(\sup _{0 \leq s \leq n}|X(s)-x|<1\right), \quad n \geq 1 .
$$

For any positive integers $n$ and $m$,

$$
\begin{aligned}
a_{n+m}= & \sup _{x \in \mathbb{R}} \mathbb{P}\left(\sup _{0 \leq s \leq n}|X(s)-x|<1 ; \sup _{n \leq s \leq n+m}|X(s)-x|<1\right) \\
= & \sup _{x \in \mathbb{R}} \mathbb{P}\left(\sup _{0 \leq s \leq n}|X(s)-x|<1 ;\right. \\
& \left.\quad \sup _{n \leq s \leq n+m}\left|\int_{n}^{s}(W(u)-W(n)) d u+X(n)+(s-n) W(n)-x\right|<1\right) .
\end{aligned}
$$

Since $\{W(v+n)-W(n) ; v \geq 0\}$ is a Brownian motion starting from 0 and is independent of $\{W(t) ; 0 \leq t \leq n\}$, we have

$$
\begin{aligned}
& a_{n+m}=\sup _{x \in \mathbb{R}} \mathbb{P}\left(\sup _{0 \leq s \leq n}|X(s)-x|<1 ;\right. \\
&\left.\sup _{0 \leq s \leq m}|\tilde{X}(s)+X(n)+s W(n)-x|<1\right),
\end{aligned}
$$


where $\{\widetilde{X}(t) ; t \geq 0\}$ stands for an independent copy of $\{X(t) ; t \geq 0\}$. By the shifted ball property for Gaussian measures (cf. Anderson [3]),

$$
\mathbb{P}\left(\sup _{0 \leq s \leq t}|X(s)-x|<\lambda\right) \leq \mathbb{P}\left(X^{*}(t)<\lambda\right),
$$

for all $t>0, \lambda>0$ and $x \in \mathbb{R}$. Conditioning on $\{W(t) ; 0 \leq t \leq n\}$ on the right-hand-side of (2.2) yields

$$
a_{n+m} \leq \mathbb{P}\left(X^{*}(n)<1\right) \mathbb{P}\left(X^{*}(m)<1\right) \leq a_{n} a_{m},
$$

which means that $\log a_{n}$ is subadditive. Hence,

$$
\lim _{n \rightarrow \infty} \frac{1}{n} \log a_{n}=\inf _{n \geq 1} \frac{1}{n} \log a_{n}
$$

exists, and clearly lies in $[-\infty, 0)$. It now suffices to observe that by means of $(2.3)$, $a_{n}$ is nothing else but $\mathbb{P}\left(X^{*}(n)<1\right)$.

Remark 2.1. That

$$
\limsup _{\varepsilon \rightarrow 0} \varepsilon^{2 / 3} \log \mathbb{P}\left(X^{*}(1)<\varepsilon\right)<0
$$

was known, cf. Stolz [29, Chap. 5] (it can also be deduced using the argument in the proof of Theorem 1.1 of Kuelbs and Li [15], for example). It is also possible, using Proposition 8 of Lachal [20], to show that

$$
\limsup _{\varepsilon \rightarrow 0} \varepsilon^{2 / 3} \log \mathbb{P}\left(X^{*}(1)<\varepsilon\right) \leq-\frac{1}{e}\left(\frac{\pi}{6}\right)^{1 / 3} .
$$

Since $(\pi / 6)^{1 / 3} / e<3 / 8$, this is not as sharp as the estimate $c_{1} \geq 3 / 8$ in Remark 1.4. However, as we have pointed out, the harder part in Theorem 1.1 is to show the finiteness of the limit. Classical techniques seem to yield only

$$
\liminf _{\varepsilon \rightarrow 0} \varepsilon^{2} \log \mathbb{P}\left(X^{*}(1)<\varepsilon\right)>-\infty,
$$

which clearly is not optimal.

2.2. Zero-one law for the lower limit in (1.5). Recall that we have proved (1.4) for some constant $c_{1} \in(0, \infty]$. Define

$$
c_{2} \triangleq \liminf _{t \rightarrow \infty} \frac{X^{*}(t)}{\varphi(t)}
$$

We next verify that $c_{2}$ is almost surely a constant lying in $[0, \infty]$.

Fix $u>0$, and let $\mathcal{T}_{u}$ be the $\sigma$-field generated by $\{W(r) ; r \geq u\}$. The tail $\sigma$-field of $W$ is then $\mathcal{T} \triangleq \cap_{u>0} \mathcal{T}_{u}$. According to Kolmogorov's $0-1$ law, $\mathcal{T}$ is trivial. It therefore suffices to show that $c_{2}$ is $\mathcal{T}_{u}$ measurable for all choices of $u>0$. Fix $u>0$ and consider the following for all $t>u$ :

$$
\begin{aligned}
X^{*}(u) & \vee\left[\sup _{u \leq s \leq t}\left|\int_{u}^{s} W(r) d r\right|-|X(u)|\right] \leq X^{*}(t) \leq X^{*}(u) \\
& \vee\left[\sup _{u \leq s \leq t}\left|\int_{u}^{s} W(r) d r\right|+|X(u)|\right] .
\end{aligned}
$$


The above is an immediate consequence of triangle inequality. Since $u>0$ is fixed and $\lim _{t \rightarrow \infty} \varphi(t)=\infty$,

$$
c_{2}=\liminf _{t \rightarrow \infty} \frac{1}{\varphi(t)} \sup _{u \leq s \leq t}\left|\int_{u}^{s} W(r) d r\right| .
$$

That is $c_{2}$ is $\mathcal{T}_{u}$-measurable. This is the desired conclusion.

2.3. Finiteness of $c_{2}$. Recalling Eq. (2.4), we propose to verify that $c_{2}<\infty$. To this end, define the local time of $W$ by,

$$
L(t, x) \triangleq \frac{d}{d x} \int_{0}^{t} \mathbb{I}_{\{W(s)<x\}} d s .
$$

According to a theorem of Trotter [32], a version of $(t, x) \mapsto L(t, x)$ can be chosen so as to be almost surely continuous. We will work only with this version. Write

$$
\tau(s)=\inf \{t>0: L(t, 0)>s\}, \quad s>0 .
$$

This is the right-continuous inverse local time process at 0 . Consider the process

$$
Y(t) \triangleq X(\tau(t)), \quad t>0 .
$$

In agreement with the notation introduced in Section 1, let

$$
Y^{*}(t) \triangleq \sup _{0<s \leq t}|Y(s)|, \quad t>0 .
$$

We claim that, with probability one, for any $t>0$,

$$
X^{*}(\tau(t))=Y^{*}(t)
$$

Assuming (2.5) for the moment, let us prove that $c_{2}<\infty$. We begin with the observation that $t \mapsto Y(t)$ is a symmetric stable Lévy process with index $(1 / 3)$. As symmetry and scaling are immediate, let us verify that $Y$ has iid increments. Since $W(\tau(t))=0$, a.s.,

$$
Y(t+s)-Y(s)=\int_{0}^{\tau(s+t)-\tau(s)}(W(r+\tau(s))-W(\tau(s))) d r .
$$

Now we just note that $t \mapsto \tau(s+t)-\tau(s)$ is the inverse local time for $r \mapsto$ $W(r+\tau(s))-W(\tau(s))$. The fact that $W$ has iid increments implies that $Y$ does as well. Theorem 11.6 of Fristedt [9] now implies that for some absolute constant $c_{3} \in(0, \infty)$,

$$
\liminf _{t \rightarrow \infty} \frac{(\log \log t)^{3}}{t^{3}} Y^{*}(t)=c_{3}, \quad \text { a.s. }
$$

On the other hand, $t \mapsto \tau(t)$ is a strictly stable subordinator with index $(1 / 2)$. For a discussion of this and more, see Chapter 5 of Fristedt [9]. Using Theorem 6.20 of [9], we see that

$$
\liminf _{t \rightarrow \infty} \frac{\tau(t)}{t^{2} / \log \log t}=\frac{1}{2}, \quad \text { a.s. }
$$

An immediate consequence of the above is that

$$
\liminf _{t \rightarrow \infty} \frac{\log \log \tau(t)}{\log \log t} \geq 1, \quad \text { a.s. }
$$


On the other hand, by Theorem 6.1 of [9], for any $\varepsilon>0$,

$$
\lim _{t \rightarrow \infty} \frac{\tau(t)}{t^{1 / 2+\varepsilon}}=0, \quad \text { a.s. }
$$

Since $\varepsilon>0$ is arbitrary,

$$
\limsup _{t \rightarrow \infty} \frac{\log \log \tau(t)}{\log \log t} \leq 1, \quad \text { a.s. }
$$

Consequently, we have the following:

$$
\lim _{t \rightarrow \infty} \frac{\log \log \tau(t)}{\log \log t}=1, \quad \text { a.s. }
$$

Assembling (2.5)-(2.8) yields

$$
\liminf _{t \rightarrow \infty} \frac{X^{*}(t)}{\varphi(t)} \leq \sqrt{8} c_{3}, \quad \text { a.s. }
$$

proving the finiteness of $c_{2}$.

It remains to show (2.5). Clearly, $X^{*}(\tau(t)) \geq Y^{*}(t)$. We now prove the other inequality. Fix $t>0$ and observe that $X$ is a differentiable process. Continuity alone suffices to show that $X^{*}(\tau(t))=X\left(t^{*}\right)$, for some $t^{*} \in[0, \tau(t)]$. If $t^{*}=0$ or $\tau(t)$, then $W\left(t^{*}\right)=0$. Otherwise $t^{*}$ lies in $(0, \tau(t))$, which means that the derivative of $X$ vanishes at $t^{*}$, i.e. $W\left(t^{*}\right)=0$. Therefore in all situations, $t^{*} \in[0, \tau(t)]$ is a zero point of $W$. According to Corollary VI.2.6 of Revuz and Yor [25], there exists some $s \in[0, t]$ such that either $t^{*}=\tau(s)$ or $t^{*}=\tau\left(s^{-}\right) \triangleq \lim _{r \rightarrow s^{-}} \tau(r)$. In either case, we see that $X^{*}(\tau(t)) \leq \sup _{0<s \leq t}|X(\tau(s))|=Y^{*}(t)$.

Thus for any given $t>0$, we have proved (2.5) (with an identity "almost surely", instead of a true identity). Since the processes on both sides of (2.5) are càdlàg, they are indistinguishable.

Remark 2.2. The exact value of the constant $c_{3}$ in Chung's LIL for $(1 / 3)$-stable Lévy processes (cf. (2.6)) is unknown. However, from (1.5), (2.9), Remark 1.4 and the relation $c_{2}=\left(c_{1}\right)^{3 / 2}$, it follows that

$$
c_{3} \geq \frac{3 \sqrt{3}}{64}
$$

2.4. Finiteness of $c_{1}$ and positivity of $c_{2}$. In this section, we wish to prove that $c_{1} \leq\left(c_{2}\right)^{2 / 3}$. In particular, we see from $\S 2.3$ that $c_{1}<\infty$ and $c_{2}>0$. We will achieve our goal by arguing by contradiction. Fix a constant $K \in\left(0, c_{1}\right)$. By (1.4), for all sufficiently small $\varepsilon>0$,

$$
\mathbb{P}\left(X^{*}(1)<\varepsilon\right) \leq \exp \left(-\frac{K}{\varepsilon^{2 / 3}}\right) .
$$

Fix $\gamma \in\left(0, K^{2 / 3}\right)$ and $R>1$. Define $t_{n} \triangleq R^{n}$. By (2.1) and (2.10), for all $n$ large,

$$
\begin{aligned}
\mathbb{P}\left(X^{*}\left(t_{n}\right)<\gamma \varphi\left(t_{n+1}\right)\right) & =\mathbb{P}\left(X^{*}(1)<\frac{\gamma \varphi\left(t_{n+1}\right)}{\left(t_{n}\right)^{3 / 2}}\right) \\
& \leq \exp \left(-K \gamma^{-2 / 3} \log \log t_{n+1}\right) \\
& =\frac{R^{-K \gamma^{-2 / 3}}}{(n+1)^{K \gamma^{-2 / 3}}},
\end{aligned}
$$


which is summable in $n$. By the Borel-Cantelli lemma, almost surely for large $n$, $X^{*}\left(t_{n}\right) \geq K \varphi\left(t_{n+1}\right)$. Let $t \in\left[t_{n}, t_{n+1}\right]$, we have

$$
X^{*}(t) \geq X^{*}\left(t_{n}\right) \geq \gamma \varphi\left(t_{n+1}\right) \geq \gamma \varphi(t)
$$

which yields

$$
\liminf _{t \rightarrow \infty} \frac{X^{*}(t)}{\varphi(t)} \geq \gamma, \quad \text { a.s. }
$$

The above would hold for arbitrary values of $\gamma<K^{2 / 3}<\left(c_{1}\right)^{2 / 3}$. Consequently, $c_{2} \geq\left(c_{1}\right)^{2 / 3}$ as desired.

2.5. Equality between $c_{2}$ and $\left(c_{1}\right)^{3 / 2}$. In view of the discussion in $\S 2.4$, it remains only to verify $c_{2} \leq\left(c_{1}\right)^{3 / 2}$.

Fix a small rational number $\varepsilon>0$. Define (recalling that we have proved $0<$ $\left.c_{1}<\infty\right)$,

$$
\begin{aligned}
& t_{n} \triangleq n^{n}, \\
& x_{n} \triangleq(1+2 \varepsilon)\left(c_{1}\right)^{3 / 2} \varphi\left(t_{n}\right), \\
& E_{n} \triangleq\left\{\sup _{t_{n-1} \leq t \leq t_{n}}\left|\int_{t_{n-1}}^{t}\left(W(u)-W\left(t_{n-1}\right)\right) d u\right|<x_{n}\right\} .
\end{aligned}
$$

By (2.1) and (1.4), and the fact that $W$ has stationary increments,

$$
\begin{aligned}
\mathbb{P}\left(E_{n}\right) & =\mathbb{P}\left(X^{*}\left(t_{n}-t_{n-1}\right)<x_{n}\right) \\
& \geq \exp \left(-(1+\varepsilon) c_{1} \frac{t_{n}-t_{n-1}}{\left(x_{n}\right)^{2 / 3}}\right) \\
& \geq \exp \left(-\log \log t_{n}\right),
\end{aligned}
$$

for all large $n$. This yields $\sum_{n} \mathbb{P}\left(E_{n}\right)=\infty$. Since the events $E_{n}$ are independent, it follows from the Borel-Cantelli lemma that

$$
\liminf _{n \rightarrow \infty} \frac{1}{\varphi\left(t_{n}\right)} \sup _{t_{n-1} \leq t \leq t_{n}}\left|\int_{t_{n-1}}^{t}\left(W(u)-W\left(t_{n-1}\right)\right) d u\right| \leq\left(c_{1}\right)^{3 / 2}, \quad \text { a.s. }
$$

On the other hand, according to the usual LIL and the definitions of $t_{n}$ and $\varphi(\cdot)$,

$$
\begin{aligned}
\lim _{n \rightarrow \infty} \frac{1}{\varphi\left(t_{n}\right)} \sup _{0 \leq t \leq t_{n-1}}\left|\int_{t_{n-1}}^{t}\left(W(u)-W\left(t_{n-1}\right)\right) d u\right| & =0, \quad \text { a.s. } \\
\lim _{n \rightarrow \infty} \frac{t_{n}\left|W\left(t_{n-1}\right)\right|}{\varphi\left(t_{n}\right)}=0, & \text { a.s. }
\end{aligned}
$$

Hence,

$$
\liminf _{n \rightarrow \infty} \frac{X^{*}\left(t_{n}\right)}{\varphi\left(t_{n}\right)} \leq\left(c_{1}\right)^{3 / 2}, \quad \text { a.s. }
$$

which implies $c_{2} \leq\left(c_{1}\right)^{3 / 2}$. 


\section{Proof of Theorem 1.3}

The proof of Theorem 1.3 is based on the following Laplace transform of the law of $\int_{0}^{1} X^{2}(s) d s$.

Theorem 3.1. For any $\theta>0$,

$$
\mathbb{E} \exp \left(-\frac{\theta^{2}}{2} \int_{0}^{1} X^{2}(s) d s\right)=\left(\frac{2}{\cosh ^{2} \sqrt{\theta / 2}+\cos ^{2} \sqrt{\theta / 2}}\right)^{1 / 2} .
$$

Assuming Theorem 3.1 for the moment, we can prove Theorem 1.3.

Proof of Theorem 1.3. It is seen from the Laplace transform (3.1) that

$$
\lim _{\lambda \rightarrow \infty} \lambda^{-1 / 4} \log \mathbb{E} \exp \left(-\lambda \int_{0}^{1} X^{2}(s) d s\right)=-2^{-1 / 4} .
$$

Eq. (1.7) follows by applying de Bruijn's exponential Tauberian theorem (cf. Bingham et al. [5, Theorem 4.12.9]) to $\alpha=-3$ and $B=(3 / 8) d^{4 / 3}$. The Chung LIL (1.8) is obtained from (1.7) using the same argument as in Sections 2.4-2.5. The details are omitted.

Before proving Theorem 3.1, we need a preliminary result.

Lemma 3.2. Let $\{\widetilde{W}(t) ; t \geq 0\}$ be an independent copy of $\{W(t) ; t \geq 0\}$. For any $\xi \in \mathbb{R}$,

$$
\mathbb{E} \exp \left(i \xi \int_{0}^{1} W(t) \widetilde{W}(1-t) d t\right)=\left(\frac{2}{\cosh ^{2} \sqrt{\xi / 2}+\cos ^{2} \sqrt{\xi / 2}}\right)^{1 / 2} .
$$

Proof of Lemma 3.2. Since

$$
\left\{\frac{W(t)+\widetilde{W}(t)}{\sqrt{2}}, \frac{W(t)-\widetilde{W}(t)}{\sqrt{2}} ; t \geq 0\right\}
$$

is an independent copy of $\{W(t), \widetilde{W}(t) ; t \geq 0\}$, we have

$$
\begin{aligned}
\int_{0}^{1} W(t) \widetilde{W}(1-t) d t \stackrel{(\mathrm{d})}{=} \frac{1}{2} \int_{0}^{1}(W(t)+\widetilde{W}(t))(W(1-t)-\widetilde{W}(1-t)) d t \\
=\frac{1}{2} \int_{0}^{1} W(t) W(1-t) d t-\frac{1}{2} \int_{0}^{1} \widetilde{W}(t) \widetilde{W}(1-t) d t .
\end{aligned}
$$

The law of $\int_{0}^{1} W(t) W(1-t) d t$ is determined by the following result due to Klyachko and Solodyannikov [12]:

$$
\mathbb{E} \exp \left(i \xi \int_{0}^{1} W(t) W(1-t) d t\right)=\left(\frac{1+i}{\cosh \sqrt{\xi}+i \cos \sqrt{\xi}}\right)^{1 / 2},
$$

from which (3.2) immediately follows with the aid of (3.3).

Proof of Theorem 3.1. Clearly, $t \mapsto X(t)$ is a centered Gaussian process. It is therefore possible to prove (3.1) using eigenvalue expansions for Gaussian quadratic functionals. However, by making use of a stochastic Fubini argument which we have learnt from Donati-Martin and Yor ([8]; see also Yor [33, Chap. II]), we can obtain 
(3.1) without any technical computations. All that we need is the introduction of an auxiliary independent Brownian motion. Indeed, we have

$$
\begin{aligned}
\mathbb{E} \exp \left(-\frac{\theta^{2}}{2} \int_{0}^{1} X^{2}(t) d t\right) & =\mathbb{E} \exp \left(i \theta \int_{0}^{1} X(t) d \widetilde{W}(t)\right) \\
& =\mathbb{E} \exp \left(i \theta \int_{0}^{1}\left(\int_{0}^{t} W(s) d s\right) d \widetilde{W}(t)\right) \\
& =\mathbb{E} \exp \left(i \theta \int_{0}^{1} W(s)(\widetilde{W}(1)-\widetilde{W}(s)) d s\right) .
\end{aligned}
$$

Since $\{\widetilde{W}(1)-\widetilde{W}(1-t) ; 0 \leq t \leq 1\}$ is Brownian motion independent of $\{W(t) ; 0 \leq$ $t \leq 1\}$, the above expression equals

$$
\mathbb{E} \exp \left(i \theta \int_{0}^{1} W(s) \widetilde{W}(1-s) d s\right)
$$

which, in light of Lemma 3.2, implies (3.1).

\section{SOME FURTHER REMARKS}

4.1. Inverse local time. The introduction of the inverse local time plays an important rôle in our study of integrated Brownian motion. The same approach may be used for Brownian motion (and other processes). For example, by the Brownian excursion theory, it is easily seen that $\left(\sup _{0 \leq u \leq \tau(t)}|W(u)|\right)^{-1}$ has an exponential law of parameter $t$; cf. Lemma 2.4 (i) of [11]. By (a careful application of) the Borel-Cantelli lemma, this leads to:

$$
\liminf _{t \rightarrow \infty} \frac{\log \log t}{t} \sup _{0 \leq u \leq \tau(t)}|W(u)|=1, \quad \text { a.s. }
$$

(For details and more general results, cf. Khoshnevisan [11] and Knight [13].) Jointly considered with (2.7)-(2.8), the estimate yields

$$
\liminf _{t \rightarrow \infty} \frac{(\log \log t)^{1 / 2}}{t^{1 / 2}} \sup _{0 \leq u \leq t}|W(u)| \leq \sqrt{2}, \quad \text { a.s. }
$$

Compared with the classical Chung [6] LIL for $W$ (which says that the liminf term in (4.1) equals $\pi / \sqrt{8}$ ), it is noted that (4.1) gives the correct rate function (but not the constant) for the lower behavior of Brownian motion.

4.2. $L^{p}$-norm. Consider the integrated Brownian motion process $X$ under the $L^{p}$-norm $\|\cdot\|_{p}$, instead of under the uniform sup-norm:

$$
\|f\|_{p} \triangleq\left(\int_{0}^{1}|f(t)|^{p} d t\right)^{1 / p}
$$

We can use the same proof as in Section 2 to obtain the following small ball estimate for $\|X\|_{p}$.

Theorem 4.1. Let $X$ be as in (1.2), and let $1 \leq p \leq \infty$. There exists a constant $c_{4}(p) \in(0, \infty)$, depending only on $p$, such that

$$
\lim _{\varepsilon \rightarrow 0} \varepsilon^{2 / 3} \log \mathbb{P}\left(\|X\|_{p}<\varepsilon\right)=-c_{4}(p) .
$$


It is obvious that $c_{4}(p)$ is nondecreasing in $p$, and that the constant $c_{1}$ in (1.4) is nothing else but $c_{4}(\infty)$. However, we do not know the exact value of $c_{4}(p)$ except for $c_{4}(2)=3 / 8$.

4.3. Integrated Brownian bridge. Let $\{\Lambda(s) ; 0 \leq s \leq 1\}$ be a standard Brownian bridge, and let

$$
Z(t) \triangleq \int_{0}^{t} \Lambda(s) d s, \quad 0 \leq t \leq 1
$$

which is a continuous centered Gaussian process. Write $Z^{*}(t) \triangleq \sup _{0 \leq u \leq t}|Z(u)|$ for brevity.

Theorem 4.2. We have,

$$
\lim _{\varepsilon \rightarrow 0} \varepsilon^{2 / 3} \log \mathbb{P}\left(Z^{*}(1)<\varepsilon\right)=-c_{1},
$$

where $c_{1} \in(0, \infty)$ is the absolute constant in (1.4).

Proof. We will show the following: for all $\delta \in(0,1)$ and $\varepsilon>0$,

$$
\mathbb{P}\left(X^{*}(1) \leq \varepsilon\right) \leq \mathbb{P}\left(Z^{*}(1) \leq \varepsilon\right) \leq \sqrt{\frac{\pi}{2}} \frac{e^{2 \varepsilon^{2} \delta^{2}}}{2 \varepsilon \delta} \mathbb{P}\left(X^{*}(1) \leq \varepsilon(1+\delta)\right) .
$$

In light of Theorem 1.1, this implies the theorem.

The Brownian bridge $\Lambda$ can be realized as $\Lambda(t)=W(t)-t W(1)$ for $0 \leq t \leq 1$, which is, moreover, independent of the variable $W(1)$. Hence,

$$
Z(t)=X(t)-\frac{t^{2}}{2} W(1), \quad 0 \leq t \leq 1
$$

By the independence of $\Lambda$ and $W(1)$,

$$
\begin{aligned}
\mathbb{P}\left(Z^{*}(1) \leq \varepsilon\right) \mathbb{P}(|W(1)| \leq 2 \varepsilon \delta) & =\mathbb{P}\left(Z^{*}(1) \leq \varepsilon,|W(1)| \leq 2 \varepsilon \delta\right) \\
& \leq \mathbb{P}\left(X^{*}(1) \leq \varepsilon(1+\delta)\right) .
\end{aligned}
$$

By the form of the Gaussian density, for all $\varepsilon>0$,

$$
\mathbb{P}(|W(1)| \leq \varepsilon) \geq \sqrt{\frac{2}{\pi}} \varepsilon e^{-\varepsilon^{2} / 2}
$$

The upper bound in (4.3) is immediate.

For the other inequality in (4.3), we use Anderson's inequality [3]. For us, it says that for any $f$,

$$
\mathbb{P}\left(\sup _{0 \leq s \leq 1}|Z(s)+f(s)| \leq \varepsilon\right) \leq \mathbb{P}\left(Z^{*}(1) \leq \varepsilon\right)
$$

Taking $f(s)=s^{2} W(1) / 2$ and using (4.4) together with the independence of $f$ and $Z$, the first inequality in (4.3) follows.

Here is an application of Theorem 4.2: let $\left\{\alpha_{n}(t) ; 0 \leq t \leq 1\right\}$ be the empirical process, based on the first $n$ observations of independent variables uniformly distributed in $(0,1)$. Define

$$
\Xi_{n}(t) \triangleq \int_{0}^{t} \alpha_{n}(s) d s, \quad 0 \leq t \leq 1
$$

As for the case of integrated Brownian motion, the small ball estimate (4.2) for the integrated Brownian bridge leads to a Chung-type LIL for the integrated Kiefer 
process, which, by means of a strong approximation, in turn yields the corresponding result for $\Xi_{n}$. We present the statement without proof.

Theorem 4.3. Let $c_{1}$ be as in (1.4). Then,

$$
\liminf _{t \rightarrow \infty} \frac{(\log \log t)^{3 / 2}}{t^{3 / 2}} \sup _{0 \leq t \leq 1}\left|\Xi_{n}(t)\right|=\left(c_{1}\right)^{3 / 2}, \quad \text { a.s. }
$$

\section{ACKNOWLEDGEMENT}

We wish to thank Jim Kuelbs and Aimé Lachal for enlightening discussions and pointing out some errors in the first draft. We also thank an anonymous referee for providing us with a number of useful remarks.

\section{REFERENCES}

[1] Albeverio, S., Hilbert, A. and Kolokoltsov, V.N. (1997). Transience of stochastically perturbed classical Hamiltonian systems and random wave operators. Stochastics Stochastics Rep. $\underline{60}$. CMP 97:09

[2] Albeverio, S., Hilbert, A. and Kolokoltsov, V.N. (1996). Estimates uniform in time for the transition probability of diffusions with small drift and for stochastically perturbed Newton equations. (preprint)

[3] Anderson, T.W. (1955). The integral of symmetric unimodular function over a symmetric convex set and some probability inequalities. Proc. Amer. Math. Soc. $\underline{6}$ 170-176. MR 16:1005a

[4] Baldi, P. and Roynette, B. (1992). Some exact equivalents for the Brownian motion in Hölder norm. Probab. Th. Rel. Fields 93 457-484. MR 94a:60117

[5] Bingham, N.H., Goldie, C.M. and Teugels, J.L. (1987). Regular Variation. Cambridge University Press, Cambridge. MR 88i:26004

[6] Chung, K.L. (1948). On the maximum partial sums of sequences of independent random variables. Trans. Amer. Math. Soc. 64 205-233. MR 10:132b

[7] Deheuvels, P. and Mason, D.M. (1996). Random fractals and Chung-type functional laws of the iterated logarithm. (preprint).

[8] Donati-Martin, C. and Yor, M. (1991). Fubini's theorem for double Wiener integrals and the variance of the Brownian path. Ann. Inst. H. Poincaré Probab. Statist. 27 181-200. MR 92m:60072

[9] Fristedt, B.E. (1974). Sample functions of stochastic processes with stationary, independent increments. Adv. Probab. $\underline{3}$ 241-396. Dekker, New York. MR 53:4240

[10] Kac, M. (1962). Probability theory: its role and its impact. SIAM Rev. 4 1-11. MR 27:1972

[11] Khoshnevisan, D. (1996). Lévy-classes and self-normalization. Electronic J. Probab. 1, paper no.1, 1-18. MR 97h:60024

[12] Klyachko, A.A. and Solodyannikov, Yu.V. (1987). Calculation of characteristic functions of some functionals of a Wiener process and a Brownian bridge (English translation). Th. Probab. Appl. 31 500-504. MR 88f:60145

[13] Knight, F.B. (1973). Local variation of diffusion in local time. Ann. Probab. 1 1026-1034. MR 53:1754

[14] Kolokoltsov, V.N. (1997). A note on the long time asymptotics of the Brownian motion with applications to the theory of quantum measurement. Potential Anal. 7 759-764. CMP 98:04

[15] Kuelbs, J. and Li, W.V. (1993). Small ball problems for Brownian motion and the Brownian sheet. J. Theoretical Probab. $\underline{6}$ 547-577. MR 94h:60121

[16] Kuelbs, J. and Li, W.V. (1993). Metric entropy and the small ball problem for Gaussian measures. J. Funct. Anal. 116 133-157. MR 94j:60078

[17] Kuelbs, J., Li, W.V. and Shao, Q.-M. (1995). Small ball probabilities for Gaussian processes with stationary increments under Hölder norms. J. Theoretical Probab. $\underline{8}$ 361-386. MR 96b:60096

[18] Kuelbs, J., Li, W.V. and Talagrand, M. (1994). Lim inf results for Gaussian samples and Chung's functional LIL. Ann. Probab. 22 1879-1903. MR 96h:60047 
[19] Lachal, A. (1992). Étude des Trajectoires de la Primitive du Mouvement Brownien. Thèse de Doctorat de l'Université Claude Bernard Lyon I.

[20] Lachal, A. (1997). First exit time from a bounded interval for integrated Brownian motion. C. R. Acad. Sci. Paris. Sér. I Math. $\underline{324}$ 559-564. MR 98a:60116

[21] Li, W.V. (1992). Lim inf results for the Wiener process and its increments under the $L_{2}-$ norm. Probab. Th. Rel. Fields 92 69-90. MR 93g:60077

[22] Li, W.V. (1992). Comparison results for the lower tail of Gaussian seminorms. J. Theoretical Probab. $\underline{5}$ 1-31. MR 93k:60088

[23] Li, W.V. and Shao, Q.-M. (1996). Small ball estimates for Gaussian processes under Sobolev type norms. (preprint)

[24] McKean, H.P. (1963). A winding problem for a resonator driven by a white noise. J. Math. Kyoto Univ. 2 227-235. MR 27:6312

[25] Revuz, D. \& Yor, M. (1994). Continuous Martingales and Brownian Motion. (2nd edition) Springer, Berlin. MR 95h:60072

[26] Rice, S.O. (1944). Mathematical analysis of random noise. Bell Syst. Techn. J. 23 282-332. MR 6:89b

[27] Shao, Q.-M. and Wang, D. (1995). Small ball probabilities of Gaussian fields. Probab. Th. Rel. Fields 102 511-517. MR 96h:60069

[28] Shi, Z. (1996). Small ball probabilities for a Wiener process under weighted sup-norms, with an application to the supremum of Bessel local times. J. Theoretical Probab. 9 915-929. MR 98b:60076

[29] Stolz, W. (1995). Mesures Gaussiennes de Petites Boules et Petites Déviations. Thèse de Doctorat de l'Université Paul Sabatier Toulouse III.

[30] Stolz, W. (1996). Some small ball probabilities for Gaussian processes under non-uniform norms. J. Theoretical Probab. 9 613-630. MR 97h:60036

[31] Talagrand, M. (1994). The small ball problem for the Brownian sheet. Ann. Probab. 22 13311354. MR 95k:60049

[32] Trotter, H.F. (1958). A property of Brownian motion paths. Illinois J. Math. 2 425-433. MR 20:2795

[33] Yor, M. (1992). Some Aspects of Brownian Motion. Part I: Some Special Functionals. Birkhäuser, Basel. MR 93i:60155

Department of Mathematics, Univeristy of Utah, Salt Lake City, Utah 82112

E-mail address: davar@math.utah.edu

L.S.T.A., Université Paris Vi, 4, Place Jussieu, 75252 Paris Cedex 05, France

E-mail address: shi@ccr.jussieu.fr 